\title{
Formación de postgrado de gestores culturales en España
}

Con el Boletín PH-3I el Departamento de Formación del Instituto Andaluz del Patrimonio Histórico inicia una nueva manera de informar sobre la oferta formativa de posgrado en España en materia de patrimonio.

En años anteriores este Departamento presentaba, en el Boletín PH de junio, la oferta de formación de las universidades españolas y otras instituciones similares relativa a cursos de posgrado de las diversas materias de interés en el patrimonio: museos, archivos, documentación, arquitectura, conservación, etcétera.

A partir de este Boletín cambiamos la manera de ofrecer esta información presentando, de forma monográfica y en números sucesivos del Boletín, la información relativa a:

I. Boletín 3 I (junio) Gestión cultural

2. Boletín 32 (septiembre) Ocio y Turismo

3. Boletín 33 (diciembre) Arquitectura y Patrimonio

4. Etc.

Cada número llevará un desarrollo más amplio de las características de los cursos que se ofertan, así como un comentario de personas relevantes en la temática e implicadas en la formación.

Esta primera entrega tratará sobre la formación de los gestores culturales en España. Uno de los retos que tiene marcada la Consejería de Cultura de la Junta de Andalucía para los próximos años es promover la puesta en marcha en Andalucía de un curso de posgrado, posiblemente un máster en políticas y gestión cultural, en cooperación con alguna universidad andaluza, para la anualidad 200 1/2002 del que ya mantendremos a nuestros lectores debidamente informados a medida que se concrete el proyecto.

\section{FORMACIÓN DE POSGRADO EN ESPAÑA EN EL ÁMBITO DE LA GESTIÓN CULTURAL}

Si algo ha caracterizado el sector de la gestión cultural en España hasta fechas muy recientes ha sido la ausencia de formación especializada en la materia. En sus inicios la demanda de formación especializada se vinculó fundamentalmente al sector de los servicios sociales, la juventud y la educación sin considerar aún la cultura como un campo de actividad laboral propio. Para encontrar las primeras ofertas formativas dirigidas a gestores y agentes culturales hemos de remitirnos a la Diputación de Barcelona quien a tra- vés de su Centro de Estudios y Recursos Culturales, CERC, organiza los primeros cursos de especialización de larga duración en España por donde han pasado gran parte de los gestores que trabajan actualmente en todas las comunidades del Estado.

Sin embargo en los últimos años han surgido un buen número de iniciativas en diferentes comunidades autónomas que vienen a paliar en parte esta carencia. Será necesario esperar que pasen unos años para poder evaluar la incidencia real de esta oferta en el mercado laboral y en la formación de los profesionales.

La figura del gestor cultural es aun bastante joven y por ello la oferta formativa en este campo no está tan estructurada como la que podemos ver en otros sectores de mayor tradición como los museos o archivos. Así se constata por ejemplo la ausencia de un corpus teórico standarizado, un curriculum formativo definido, así como la escasez de bibliografía especializada en lengua castellana.

Fruto de esto es el hecho de que en los cursos de posgrado se observa una diversidad y en ocasiones indefinición en los temarios que varía de los generalistas a los especializados y que ofrece un abanico de posibilidades que oscila entre aquellos que se orientan a la gestión cultural en el ámbito local, la gestión de las artes escénicas, la gestión y conservación del patrimonio, unos que ponen mayor énfasis en el proyecto, otros que se especializan en la gestión de recursos y las habilidades directivas, los que priman la componente internacional, etc,

Es por ello que han surgido iniciativas para intentar definir el perfil y las competencias clave del gestor cultural, como el proyecto Format. El proyecto Format tiene como objetivo reflexionar sobre la experiencia de formación de gestores culturales en España y estimular el cambio de perspectiva de los agentes culturales hacia una mayor internacionalización de los proyectos creativos y de gestión, definiendo las competencias clave del gestor y proponiendo módulos formativos. El proyecto Format ha diseñado una interesante página web donde podemos encontrar abundante información relacionada con el tema de la formación y los gestores culturales, incluyendo instituciones y documentos de interés, referencias bibliográficas, redes, asociaciones, etc... http://www.interarts.net/format

En este sentido es necesario señalar el papel que a nivel internacional realiza la Red Encact de formación en gestión cultural. La Red Europea de centros de formación de administradores culturales es una red independiente de instituciones de formación y 
de personas implicadas en la formación en gestión cultural y sectores relacionados. Su objetivo es desarrollar la formación de administradores culturales en Europa y la cooperación entre los centros que organizan estas formaciones. Desde hace varios años publican un directorio de formación en administración cultural en Europa, que cuenta con la colaboración del Consejo de Europa y que se actualiza anualmente en su edición electrónica. http://www.artecnet.com/encatc/. Correo: encatc@artecnet.com

A nivel internacional destaca igualmente el Certificado europeo en administración de proyectos culturales, programa paneuropeo de formación organizado por la Fundación Marcel Hicter con sede en Bruselas y auspiciado por organismos internacionales como el Consejo de Europa y la Unesco. Para el curso 20002001 se convoca su $10^{a}$ edición y es en estos momentos el diploma internacional en la materia de mayor prestigio en Europa. Para mayor información: fond.hicter@glo.be

Para la elaboración de este número monográfico hemos pedido la colaboración de algunas personas que pensamos podían aportar diferentes y complementarias visiones sobre el tema que nos ocupa. Por un lado Alfons Martinell quien ha sido director durante varios años de los posgrados en Gestión y Políticas Culturales organizados por el Centro de Estudios y Recursos Culturales de la Diputación de Barcelona, CERC y en los dos últimos años ha sido coordinador del proyecto Format de la Iniciativa Comunitaria ADAPT, sobre formación de los gestores culturales en España. Por otro lado nos ha enviado su colaboración Francisco Zamora, Presidente de la Asociación Española de Gestores de Patrimonio Cultural. Por último hemos contado con la aportación de Javier Rodríguez Fito, Presidente de la Asociación de Gestores Culturales de Andalucía. Desde aquí agradecemos a todos ellos su colaboración.

Presentamos a continuación el listado de los cursos especializados sobre gestión cultural y del patrimonio. En estos momentos ya están saliendo a la luz las primeras iniciativas para ofertar la formación en gestión cultural como licenciatura de primer y segundo ciclo. Nosotros para este número sólo hemos considerado la oferta de posgrado o tercer ciclo. Hemos intentando recopilar aquí el conjunto de oferta formativa en la materia existente en estos momentos en todo el territorio nacional. Hay que señalar que se han incluido unicamente los cursos de posgrado de más de 150 horas y aquellos que a la hora de cierre de esta edición podían confirmar la continuidad de los mismos. Agradecemos a las personas y entidades que nos han enviado puntualmente la documentación y pedimos disculpas a los que por una razón u otra no hemos podido incluir rogándoles nos envíen la información para presentarla en posteriores ediciones y en nuestra página web donde dejaremos colgada esta información.
En una primera valoración hemos de señalar el elevado número de cursos y máster de posgrados que se ofertan en estos momentos en España. Este crecimiento de la oferta nos lleva a pensar en un aumento de la demanda por parte tanto de recién licenciados que acceden a un mercado laboral donde la especialización es cada vez más necesaria, como de los profesionales que necesitan el perfeccionamiento y reciclaje de sus conocimientos.

Junto a la ficha de cada uno de los cursos hemos destacado el número de ediciones de los mismos ya que es un indicador que nos habla de la continuidad de las iniciativas y nos ha llevado a no considerar aquellas que han sido propuestas aisladas.

En cuanto a la distribución por el territorio nacional podemos decir que la oferta se concentra en las comunidades de Cataluña, Castilla - León, Galicia, Madrid, País Vasco y Valencia. Por el momento no existe formación de este tipo en Andalucía aunque ha habido alguna iniciativa aislada como la que en su día puso en marcha la Universidad de Cádiz.

\section{LISTADO DE CURSOS DE POSTGRADO EN GESTIÓN CULTURAL Y DEL PATRIMONIO EN ESPAÑA}

\section{DIPLOMA DE POSTGRADO EN GESTIÓN Y POLÍTICAS} CULTURALES. UNIVERSIDAD DE BARCELONA.

Tiene su origen en los Seminarios de Dirección de Proyectos Culturales que de forma pionera organizó el Cerc de la Diputación de Barcelona hace unos 13 años. En su formato actual de Diploma de Posgrado están realizando la $9^{a}$ edición. La media de alumnos ha sido de unos 3035 por edición, superando en general la demanda a las plazas ofertadas. La preinscripción para la próxima edición estará abierta hasta julio de 2000. Un 10\%-20\% de los alumnos proceden de Iberoámerica. El formato concentrado permite la asistencia de alumnos de todo el territorio nacional y de Iberoamérica.

Organiza: Universidad de Barcelona y el Centro de Estudios y Recursos Culturales, Diputación de Barcelona. Director: Lluís Bonet. Coordinador académico: David Roselló.

Dirigido: Licenciados/as universitarios/as y, eventualmente, diplomados/as en materias afines a las del curso que acrediten experiencia en la gestión de servicios o proyectos culturales.

Duración: 20 créditos. Un año académico distribuido en diversos módulos formativos.

Contenido: La cultura como factor de desarrollo; Fundamentos y estrategias de las políticas culturales; Políticas culturales en Europa; Modelos de gestión e intervención cultural; Bases jurídicas del sector cultural; Bases económicas del sector cultural; Dimensiones de 
la cultura y de las artes; Gestión y organizaciones del sector cultural; Planificación, programación y evaluación; Metodología de la elaboración de proyectos culturales; Análisis de organizaciones y proyectos de gestión cultural; Elaboración de un proyecto de gestión.

Información: Centro de Estudios y Recursos Culturales. Diputación de Barcelona.

Carrer de Montalegre, 7.

0800 I Barcelona

Tel: (93) 402.25.69.

Fax: (93) 402.25.77.

Página web: www.ub.es/cultural/gchome.htm.

Correo: cultura@eco.ub.es

\section{MASTER EN GESTIÓN CULTURAL. UNIVERSIDAD DE BARCELONA.}

Al igual que el anterior este Máster recoge la experiencia de los cursos de posgrado que desde hace varios años organiza la Diputación de Barcelona. En su formato actual de Máster están en la $6^{\circ}$ edición. Se convoca cada dos años. La próxima convocatoria será en otoño del 200I, con las preinscripciones en mayo-junio del 2001.

El formato extensivo de este Máster con clases varias tardes cada semana, hace que la mayor parte de los alumnos procedan de Barcelona y su entomo. Desde hace uno o dos años el Máster ofrece una modalidad a distancia que permite el seguimiento tanto de alumnos de todo el territorio nacional como internacional. El Máster a distancia se convoca cada año. El período de preinscripción se cierra el 30 de septiembre aproximadamente.

Organiza: Universidad de Barcelona y el Centro de Estudios y Recursos Culturales, Diputación de Barcelona. Director: Lluís Bonet. Coordinador académico: Xavier Santajuliana.

Dirigido: Licenciados/as universitarios/as.

Duración: 2 años.

\section{Contenido:}

I. Fundamentos teóricos y prácticos

Se compone de siete cursos de carácter introductorio (de los que hay que elegir cinco) con el objetivo de dotar a los alumnos de información, vocabulario y técnicas de gestión básicas.

II. La Gestión cultural

La segunda parte del programa se centra en los marcos de intervención de la gestión, las políticas y los aspectos económicos y jurídicos de la gestión cultural. Estos aspectos más teóricos se complementan con visitas, el análisis de organizaciones culturales y un viaje de estudios. Al final de esta parte se obtiene una primera titulación: el Diploma de Postgrado en Gestión Cultural.

III. Especialización

El segundo año del programa combina dos tipologías de cursos, unos de especialización y otros de instrumentos al servicio de la gestión. El curso se completa con la realización de un trabajo en prácticas en una institución cultural, una estancia en uno de los países con los se ha firmado un acuerdo de intercambio o con la realización de un trabajo de investigación.

Información: Centro de Estudios y Recursos Culturales. Diputación de Barcelona.

Carrer de Montalegre, 7.

0800 I Barcelona.

Tel: (93) 402.25.69.

Fax: (93) 402.25.77.

Página web: www.ub.es/cultural/gchome.htm.

Correo: cultura@eco.ub.es

\section{MÁSTER EN GESTIÓN CULTURAL: TURISMO, PATRIMONIO Y NATURALEZA. INSTITUTO UNIVERSITARIO ORTEGA Y GASSET. UNIVERSIDAD COMPLUTENSE.}

Están realizando en estos momentos la $5^{a}$ edición. En otoño saldrá la $6^{a}$ convocatoria. Hasta ahora la media ha sido de unos 30 alumnos por edición. La preinscripción par el próximo curso estará abierta hasta el 7 de julio de 2000. En caso de quedar plazas libres se abriría un segundo período de inscripción hasta el 22 de septiembre de 2000.

Organiza: Instituto Universitario Ortega y Gasset. Directora: Agueda Esteban Talaya. Coordinador: Xavier Esparza San Juán

Dirigido: Todas aquellas personas con formación superior interesadas en la orientación profesional hacia la gestión cultural: licenciados/as, estudiantes de postgrado, empresarios/as, profesionales independientes, personal de administraciones públicas, de instituciones privadas, formadores/as y profesores/as, miembros de asociaciones, consultores, etc.

Duración: 420 horas.

Contenido:

Introducción. Módulo I. Fundamentos de gestión cultural. Módulo II. Instrumentos operativos de la gestión cultural. Módulo III. Gestión del Patrimonio Cultural. Módulo IV. Gestión del Patrimonio Natural. Módulo V. Gestión turística del patrimonio. Módulo VI. Gestión cultural integral.

Para la obtención del título es imprescindible la realización y defensa de un Proyecto de Gestión.

Prácticas: Los alumnos del Máster podrán realizar prácticas con diferentes instituciones del ámbito cultural, patrimonial, turístico y de medio ambiente, con una duración mínima de un mes.

Información: Instituto Universitario Ortega y Gasset. C/ Fortuny, 53.

28010 Madrid.

Tel: 9 I/ 700.4I.48.

Fax: $91 /$ 700.35.30.

Correo electrónico: fogescul@accessnet.es 
MÁSTER EN GESTIÓN DEL PATRIMONIO CULTURAL. UNIVERSIDAD COMPLUTENSE.

En estos momentos realizan la $5^{a}$ edición. La próxima convocatoria saldrá en diciembre de 2000. La preinscripción está abierta en estos momentos y el plazo de matrícula es del 1 al 20 de julio. El número de alumnos es de unos 30 por edición.

Organiza: Centro Superior de Estudios de Gestión, Universidad Complutense de Madrid. Directora: Rosa Garcerán. Coordinación: Elena Jiménez

Dirigido: Licenciados/as universitarios/as.

Duración: 2 semestres.

Contenido: Módulo Histórico-Artístico. Módulo Social. Módulo Político. Módulo Instrumental. Módulo Metodológico. Proyecto Final.

Se ofrece la posibilidad de realizar un módulo con obtención de diploma en el Centre of Environmental Interpretation - CEI - (Manchester Metropolitan University).

Información: Centro Superior de Estudios de Gestión, Análisis y Evaluación.

Edificio B, Mas Ferré,

Campus de Somosaguas,

28223 Madrid.

Tel: $91 / 394.29 .50 / 57$.

Fax: $91 / 394.29 .56$.

MÁSTER EN GESTIÓN CULTURAL: MÚSICA, TEATRO Y DANZA. INSTITUTO COMPLUTENSE DE CIENCIAS MUSICALES DE MADRID. UNIVERSIDAD COMPLUTENSE.

Actualmente realizan la $6^{\circ}$ edición. La próxima convocatoria es la del 200I, siendo la preinscripción en abril y junio del 2001 y comenzando las clases en octubre del 2001. Es el único en España en su especialidad y recibe por ello gran cantidad de solicitudes. La demanda está en unos 140 por convocatoria y el $n^{\circ}$ de alumnos admitidos es de 40.

Organiza: Departamento de Gestión Cultural del Instituto Complutense de Ciencias Musicales de Madrid. Director: Emilio Casares Rodicio. Coordinadora: Nereida Fonseca.

Dirigido: Licenciados/as universitarios/as, titulados/as superiores o titulación equivalente y estudiantes del último curso de licenciatura. Excepcionalmente se admitirán diplomados/as universitarios/as de materias afines o profesionales que acrediten una notable experiencia en el campo de la gestión cultural.

Duración: 550 horas lectivas y 360 horas de práctica.
Contenido: Área de gestión cultural. Area de organización y dirección de empresas. Area de gestión estratégica de la imagen Area de derecho. Area de infraestructura y técnica escénica. Area de teoría e historia: repertorio y programación artística.

Información: Instituto Complutense de Ciencias Musicales de Madrid.

C/ Fernando VI, 4,

28004 Madrid.

Télf.: 913192710.

Fax: 913499710.

Correo electrónico: nfonseca@sgae.es.

Página web: www.sgae.es/iccmu

DIPLOMA DE ESPECIALIDAD EN GESTIÓN DE RECURSOS CULTURALES Y DIPLOMA DE EXPERTO EN GESTIÓN DE RECURSOS CUTURALES. UNIVERSIDAD DE DEUSTO-SAN SEBASTIÁN.

Están realizando la $10^{a}$ convocatoria. Para el próximo curso sólo se convoca el Diploma de Experto. La demanda varía mucho siendo la media de unos 25 alumnos. La incidencia es principalmente regional asistiendo alumnos del País vasco, la Rioja y Navarra.

Organiza: Universidad de Deusto-San Sebastián, Facultad de Filosofía y Letras.

Dirigido: a profesionales de la gestión cultural que deseen introducir criterios racionales de gestión en sus actividades cotidianas. Se requiere diploma universitario para acceder al "Diploma de Especialidad" y licenciatura universitaria para acceder al "Experto".

Duración: I curso académico.

\section{Contenido:}

I. Curso de iniciación en función de la titulación y currícula del alumno (15c.)

2. Materias troncales (28c.): marco general de la gestión y políticas culturales; empresa cultural; administración pública; marketing de servicios y comunicación; informática aplicada a la dirección; financiación cultural.

3. Proyecto cultural (15c.): gestión y dirección de proyectos culturales; seminarios de apoyo científico al proyecto.

4. Prácticas (350horas).

5. Conferencias, mesas redondas, visitas a centros culturales y otros lugares de interés.

Información: Universidad de Deusto-San Sebastián. Facultad de Filosofía y Letras.

Mundaiz 50.

Apartado 1.359

E 20080 San Sebastián.

Teléf.: 943273100 ,

ext.: 288.

Fax: 943292635. 
MÁSTER EN PROYECTOS Y COOPERACIÓN CULTURAL INTERNACIONAL. UNIVERSIDAD DE BARCELONA.

Posgrado con clara vocación internacional, se viene ofertando desde hace 5 ediciones por el Observatorio Interarts. El formato intensivo permite la asistencia de alumnos de todo el territorio nacional. Como parte del curso se programa un viaje de estudios para conocer diferentes organismos e instituciones internacionales.

Organiza: Universidad de Barcelona, Fundación Bosch i Gimpera y Observatorio Interarts. Director: Eduard Delgado Clavera

Dirigido: Titulados/as superiores y diplomados/as universitarios/as de diferentes disciplinas interesados en especializarse en cooperación cultural internacional.

Duración: El curso se realizará en un período presencial obligatorio de cuatro semanas intensivas. En una de ellas se realizará un viaje de estudio de contacto con las instituciones europeas.

\section{Contenido:}

Primer curso.

Elementos y conceptos fundamentales sobre la gestión de la cultura en nuestra sociedad. Políticas culturales. Cooperación cultural en el contexto de un mundo de globalización. Geopolítica cultural. Cooperación cultural y procesos de desarrollo.

Marco jurídico e institucional. Agentes, redes y recursos para la cooperación cultural. El proyecto como metodología para la gestión de la cooperación cultural. Análisis de los casos y experiencias significativas. Conocimiento institucional. Trabajos de síntesis de los diferentes módulos.

\section{Segundo curso}

Módulos de cooperación cultural en diferentes espacios geopolíticos. Seminarios especializados en diferentes aspectos de la cooperación internacional en el ámbito cultural y otros ámbitos. Análisis de casos y experiencias significativas. Conocimiento institucional. Elaboración de un proyecto de cooperación cultural internacional.

Información: Clara Fontdevilla. Observatorio Interarts, Rambla de Cataluña 8I pral.

08008 Barcelona.

Télf:: 934877022

Fax: 934872644 .

Correo electrónico: info@interarts.net.

Página web: www.interarts.net

\section{DIPLOMA DE POSTGRADO EN COOPERACIÓN CULTURAL IBEROAMERICANA. UNIVERSIDAD DE BARCELONA.}

Curso muy especializado en la cooperación cultural con el espacio Iberoamericano. Se realiza en formato super intensivo y a el acuden cada año un buen número de profesionales de todos los países de América del Sur.
Organiza: Universidad de Barcelona. Fundación Bosch i Gimpera. Director: Lluís Bonet.

Dirigido: Licenciados/as y profesionales de Europa y América, interesados en trabajar en proyectos de cooperación cultural en el ámbito iberoamericano. Los candidatos/as deben poseer un título universitario convalidable en España.

Duración: 20 créditos. Distribuido en un primer período presencial de 5 semanas y un segundo en el que se realizará un proyecto tutorizado.

\section{Contenido:}

A. Bases conceptuales.

Marcos institucionales de la cultura en lberoamérica. Cultura y desarrollo. Producción y mercados culturales en el espacio iberoamericano.

B. Estrategias de la cooperación cultural.

Marco jurídico de la cooperación cultural internacional. Estrategias, proyectos y recursos al servicio de la cooperación cultural internacional. Planificación, gestión y evaluación de proyectos culturales. C. Instrumentos para el diseño y gestión de proyectos de cooperación cultural. Metodología para el diseño de proyectos. Recursos técnicos para la gestión de proyectos. Diseño de un proyecto de cooperación cultural.

Información: Secretaría de los cursos de postgrado en Gestión Cultural de la Universidad de Barcelona. Muntsa Quintilla. C/ Montalegre, 7 0800 I Barcelona.

Télf.: 934022569

Fax: 934022577

Correo electrónico: cultura@eco.ub.es

Página Web: www.ub.es/cultura/GChome.htm

\section{DIPLOMA DE POSTGRADO EN DIRECCIÓN Y GESTIÓN DE INSTITUCIONES, EMPRESAS Y PLATAFORMAS CULTURALES. UNIVERSIDAD POMPEU FABRA.}

Están realizando la segunda edición y ya han convocado la tercera. El año pasado tuvo gran demanda. El cupo estaba previsto en unos 30 y se amplió a 35 alumnos. El próximo curso comienza en octubre y la preinscripción está abierta hasta el 14 de julio.

Organiza: Instituto de Educación Contínua, Universidad Pompeu Fabra. Director: Ferrán Mascarell Canalda, Ayuntamiento de Barcelona.

Dirigido: Diplomados/as o licenciados/as universitarios/as, tanto de carreras de humanidades como de economía o de ciencias, que deseen especializarse en la gestión y dirección de las organizaciones culturales y adquirir las habilidades necesarias para desarrollar tareas directivas en este terreno.

Duración: 200 horas. 
Contenido: La sociología del hecho cultural. La problemática de las instituciones, las plataformas y las empresas culturales en la sociedad actual. Estructura del sector cultural en Cataluña. La planificación estratégica. Las habilidades directivas. El marco institucional y jurídico. La gestión económica y financiera. La gestión de los recursos humanos. La gestión comercial, el marketing y la comunicación.

Información y Matrícula: Institut d'Educació Contínua. Marc Aureli, 22-36.

08006 Barcelona.

Tel: 93/542.18.06.

Fax: 93/ 542.18.08.

Página web: www.upf.es/idec Correo: idec@upf.es

MÁSTER DE GESTIÓN DE SERVICIOS CULTURALES EN LAS ADMINISTRACIONES LOCALES. UNIVERSIDAD DE VALENCIA.

El Servicio de Asistencia y Recursos Culturales, SARC, de la Diputación de Valencia viene ofertando desde hace más de 10 años diferentes cursos y seminarios para la formación y reciclaje de los profesionales de la cultura que trabajan fundamentalmente en la administración local. Este curso con formato de Máster en colaboración con la Universidad de Valencia está en su $4^{a}$ edición. La convocatoria es bianual y la próxima será en el 2001. El número de alumnos está en torno a los 30 .

Organiza: Servicio de Asistencia y Recursos Culturales - SARC- de la Diputación de Valencia. Instituto Universitario de Gestión Empresarial de la Universitat de Valencia (Estudi General). Director: D. Ismael Quintanilla Pardo. Coordinador: Francisco Molina

Dirigido: Licenciados/as o diplomados/as oficiales de cualquier universidad, valorándose especialmente los posibles años de experiencia en puesto similar

Duración: 774 horas en dos años lectivos.

\section{Contenido:}

Bloque I: Elementos Básicos de Gestión, Estrategias para la materia de Cultura.

Bloque II: Gestión Cultural Estratégica; Conceptos, Habilidades Directivas, y Herramientas de Trabajo. Bloque III: Experiencias y Prácticas.

Bloque IV: Elementos Internos del Curso.

Información: SARC, Servicio de Asistencia y Recursos Culturales.

Plaza Manises, 6.

Palau Scala.

46003 Valencia.

Tel: 96/35। 0663

Fax: 96/388.28.80.

Página web: www.adeit.uv.es
MÁSTER EN CONSERVACIÓN Y GESTIÓN DEL PATRIMONIO CULTURAL. UNIVERSIDAD DE VALENCIA.

EL Máster en Gestión y Conservación del Patrimonio se organiza en dos módulos que pueden realizarse de forma independiente, optando al Diploma de Posgrado en Gestión del Patrimonio o al Diploma en Conservación. De septiembre a diciembre se realiza el módulo de gestión. La preinscripción para este módulo está abierta hasta el 15 de julio de 2000. La media de alumnos es de unos 25 por edición. Al principio el máster duraba un año, de enero a diciembre y se hacía el programa completo. La demanda llevó a estructurarlo en dos módulos independientes. Esta reforma se hizo hace dos años y por tanto ahora están en la $2^{a}$ edición de los diplomas de posgrados y en la $5^{a}$ del Máster.

Organiza: Area de Conservación del Patrimonio Cultural. Fundación Universidad-Empresa de Valencia. Director: Daniel Benito Goerlich. Coordinador: Norberto Piqueras.

Dirigido: Licenciados/as interesados/as en la gestión y conservación del patrimonio cultural.

Duración: 450 horas.

Contenido: Pintura: procesos de degradación y criterios de conservación - restauración. Escultura: procesos de degradación y criterios de conservación restauración. El patrimonio documental. Criterios de conservación. Teoría e Historia de la restauración conservación. Metodología de la investigación arqueológica. Modelos de intervención en arquitectura. Heurística: informes y proyectos. Legislación del Patrimonio Histórico Cultural. Valor y uso del patrimonio: proyectos. Gestión del proyecto arquitectónico y arqueológico. Gestión de los bienes inmuebles. El museo: proyectos y gestión. Cultura de la imagen. Prácticum de patrimonio cultural: cursostalleres sobre técnicas artísticas, conservación preventiva y gestión del patrimonio. Visitas prácticas: museos, intervenciones arquitectónicas, yacimientos e instituciones culturales. Proyecto tutorizado.

Información: Area de Conservación del Patrimonio. Universidad de Valencia.

P/ Horno de San Nicolás n4.

4600 I Valencia.

Tel: $96 / 386.41 .29$

Fax: 96/386.46.20.

Correo electrónico: Norberto.Piqueras.@uv.es

DIPLOMA DE GESTIÓN EN BIENES CULTURALES. UNIVERSIDAD DE VALENCIA.

Módulo del Máster en Conservación y Gestión del Patrimonio que puede realizarse de forma independiente. Están realizando la segunda edición. 
Organiza: Area de Conservación del Patrimonio Cultural. Fundación Universidad- Empresa de Valencia. Director: Daniel Benito Goerlich. Coordinador: Norberto Piqueras.

Dirigido: Licenciados/as interesados/as en la gestión y conservación del patrimonio cultural.

Duración: 225 horas.

Contenido: Heurística: informes y proyectos. Legislación del Patrimonio Histórico Cultural. Valor y uso del patrimonio: proyectos. Gestión del proyecto arquitectónico y arqueológico. Gestión de los bienes inmuebles. El museo: proyectos y gestión. Cultura de la imagen.

Información: Area de Conservación del Patrimonio. Universidad de Valencia.

P/ Horno de San Nicolás n 4

4600 I Valencia.

Tel: $96 / 316.60 .44$

Fax: $96 / 386.46 .20$

Correo electrónico: Norberto.Piqueras.@uv.es

\section{MÁSTER EN GESTIÓN DEL PATRIMONIO HISTÓRICO. UNIVERSIDAD DE SALAMANCA}

Este Máster inició su andadura como título de experto, realizando dos ediciones para convertirse luego en máster. El año pasado no se convocó. La próxima convocatoria se realizará entre julio y septiembre del 2000 y será la segunda edición del Máster. Se venía realizando en Zamora y ahora probablemente pase a Salamanca.

Organiza: Universidad de Salamanca en colaboración con la fundación Rei Alfonso Henriques. Director: Luis Serrano Piedecasas Fernández.

Dirigido: Preferentemente a licenciados/as o profesionales españoles, portugueses o iberoamericanos, que hayan cursado estudios superiores o estén en posesión de un título académico homologable a una licenciatura de: Bellas Artes, Geografía, Historia, Historia del Arte o Humanidades, así como estudiantes de Arquitectura, Economía o Derecho.

Duración: 688 horas.

Contenido: Análisis del patrimonio artístico. Características del patrimonio arqueológico y etnográfico. El patrimonio histórico inmueble. Procesos de evaluación y documentación en patrimonio. Gestión del patrimonio. Rentabilización social de patrimonio.

Información: Secretaría de Tercer Ciclo y Títulos Propios de la Universidad de Salamanca.

Patio de Escuelas 3, 2.

37008 Salamanca.
Télef.: 923294 400, ext.: I |73/|442.

Fax: 923294684.

Correo electrónico: tpropios@gugu.usal.es

MÁSTER EN GESTIÓN DE BIENES CULTURALES Y PATRIMONIO. UNIVERSIDAD DE LA CORUÑA.

Organiza: Facultad de Humanidades de la Universidad de la Coruña. Director: José Antonio Fernández de Rota. Coordinación: Víctor Alonso, Pedro Armas, Bernardo Castelo y José Leira López.

Dirigido: Licenciados/as universitarios/as y preferentemente aquellos cuya especialidad curricular esté relacionada con temas de patrimonio.

Duración: 60 Créditos, con 480 horas teóricas y 120 prácticas, impartidas a lo largo de dos cursos.

\section{Contenido:}

Curso $I$

I. Teoría e historia del patrimonio.

2. Políticas culturales y legislación.

3. Enfoques actuales del patrimonio y tradiciones disciplinarias. 3.I Patrimonio histórico y arqueológico (Archivos y bibliotecas). 3.2 Patrimonio artístico y monumental. 3.3 Patrimonio etnográfico y geográfico.

4. Museología.

5. Mecenazgo, patrimonio y empresa.

6. Patrimonio y turismo cultural.

7. Practicum I (elaboración de trabajos, visitas a centros de interés, prácticas en museos...).

8. Conferencias, seminarios y jornadas.

Curso II

I. Planificación y gestión del patrimonio.

2. Patrimonio como dinámica social y mercado.

3. Documentación y gestión de las colecciones.

4. Teorías y técnicas de conservación y restauración.

5. Antigüedades.

6. Museografía.

7. Difusión y didáctica del patrimonio.

8. Practicum II (elaboración de trabajos, visitas de interés, prácticas en museos...)

9. Conferencias, seminarios y jornadas.

Información: Secretaría de la Facultad de Humanidades. Campus de Esteiro.

C/ Dr. Vázquez cabrera, s/n.

I 5403 Ferrol, La Coruña.

Tel: 98I/337400

Fax: 98I/ 337430 . 


\section{ADAPTABILIDAD DE LA FORMACION DE GES- TORES CULTURALES A NUEVOS ESCENARIOS}

Alfons Martinell Sempere

Profesor de la Universidad de Girona Presidente de Interarts

Como todas las nuevas profesiones, que han surgido fruto de los cambios en la sociedad contemporánea, la gestión cultural se ha encontrado con la necesidad de demostrar su especificidad y proveer de suficientes recursos para su reconocimiento social. Me sería difícil afirmar con contundencia que la gestión cultural ha llegado a su plenitud como profesión con suficiente diferenciación con otras funciones profesionales. Pero podemos evidenciar su evolución y extensión desde los primeros cursos formativos de la década de los ochenta.

La formación en gestión cultural se configura como respuesta a una demanda de capacitación de un gran número de profesionales que se incorporaron profesionalmente a las organizaciones e instituciones públicas culturales. En un primer período la formación respondía a las necesidades de un amplio sector de personas que habían llegado a la cultura desde funciones y disciplinas muy variadas. En esta fase la mayoría de empleos en la gestión cultural procedían de las nuevas estructuras de las administraciones públicas en sus diferentes niveles.

En la década de los noventa se produce una mayor diversificación del mercado laboral de la gestión cultural. Existe una demanda de mayor especialización, se desarrollan otros agentes ( privados y asociativos) y empiezan a observarse cambios en las tendencias habituales en la línea de un mayor crecimiento y diversificación. Pero el aspecto más destacable se observa en el lento proceso de visualización del mundo de la cultura como un sector en todas sus dimensiones. La cultura adquiere una dimensión económica, genera empleo, aporta plusvalías a otras actividades ( turismo, ocio, etc...) y crece la percepción de su importancia como de la necesidad de no abandonarla a una actividad marginal u ornamental. Estas tendencias, desgraciadamente poco asumidas por los poderes públicos, presentan una nueva visión de la gestión cultural evidenciando la importancia de una capacitación de acuerdo con estos nuevos escenarios. Algunas investigaciones, estadísticas y datos permiten evidenciar que nos encontramos ante un sector de difícil delimitación, con grandes interacciones con otras actividades y con un potencial social considerable. El cual adquiere más significado en los procesos de globalización y ante los cambios de la sociedad de la información.

En este contexto la formación en gestión cultural sigue con sus planteamientos y va generando, en España, una oferta que se puede caracterizar por:

- Tendencia a un aumento de ofertas formativas dentro del nivel de formación de postgrado universitario
- Predominio de las especialidades en el ámbito del patrimonio y poca oferta en otros sectores de la cultura

- La formación genérica en gestión cultural sigue con sus orientaciones poco definidas entre una formación básicamente técnica con poca diferenciación de otros campos de la gestión

- Falta de un reconocimiento u homologación de la formación que permita ayudar a estructurar el sector

- Cambio de los perfiles de los alumnos; de una mayoría de profesionales en ejercicio en búsqueda de capacitación a un aumento considerable de postgraduados jóvenes en disciplinas de las ciencias sociales que buscan el sector cultural una posibilidad de empleo afín a sus aspiraciones.

- Ausencia de sistemas de interlocución, redes de centros de formación, encuentros que sirvan para ayudar a una mayor consolidación de la formación y unos acuerdos mínimos entre todas las ofertas de formación. Así como la definición de unos mínimos identificables de las titulaciones propuestas.

Me atrevería a afirmar que el sector de la cultura se encuentra en buen momento si lo relacionamos con su situación de hace unos años. Su centralidad y atención ha aumentado considerablemente en los últimos años y encontramos que la mayoría de organismos internacionales (Banco Mundial, Unión Europea, etc...) empiezan a tratar el tema de la cultura en el marco de sus reflexiones. Aunque la preocupación política por el campo aún se mueve en planteamientos denostados y muy clásicos.

Ante estos escenarios nos atreveríamos a proponer algunas reflexiones sobre el futuro de la formación de gestores culturales:

a) A pesar de la necesidad de incorporar contenidos y técnicas empresariales en la formación de gestores culturales observamos con cierta preocupación un exceso de gerencialismo genérico sin adaptarse a las particularidades de la gestión de la cultura. Esta se mueve entre la eficacia y eficiencia de los medios y recursos como de la sensibilidad de relación con el sector creativo y la realidad social. Una parte importante de la cultura se mueve en el campo de las tendencias puras de la producción de bienes y adaptación al mercado, pero otro campo se encuentra en la gestión de intangibles y valores sociales y políticos, difícilmente adaptables a las lógicas clásicas de las aportaciones de la teorías de las organizaciones productivas. En este sentido abogamos por la búsqueda de unos contenidos propios que diferencien la gestión de la cultura de la gestión de otros ámbitos. 
b) La gestión de la cultura requiere una capacidad de entender las tendencias y prospectivas de las nuevas fundamentaciones de las políticas culturales. En este sentido es importante incorporar las investigaciones sobre políticas culturales como un contenido básico en la formación de gestores culturales. Esta dimensión ha de encontrar su orientación en las nuevas reflexiones de los estudios culturales, las repercusiones de los cambios de la sociedad de la información, las aportaciones de los organismos internacionales ( Consejo de Europa, Unesco, Comunidad Europea, BID, etc....) en las que proponen nuevos objetivos para las políticas culturales de los diferentes agentes culturales

c) Proponer una formación con más contenidos en el desarrollo de proyectos emprendedores en el campo de la microempresa, la iniciativa social y el trabajo de profesionales autónomos. Es decir una perspectiva empresarial adaptada a los contenidos y trabajos creativos, por ser este un sector de crecimiento y creación de empleo muy importante en Europa.

d) La gestión de la cultura ya no se puede entender sino es en relación con otros sectores ( educación, desarrollo local, turismo, ocio, etc....). La capacidad de interlocución con las lógicas multisectoriales ha de convertirse en una de las competencias claves de los profesionales de la cultura. En este sentido hemos de trabajar en una formación abierta al diálogo multidisciplinar y con capacidad de mediación y negociación con otros sectores sociales.

e) Y por último, en el marco de los cambios de la era de la información, lo formación ha de promover la adaptabilidad a la necesaria internacionalización de la gestión y el funcionamiento de las organizaciones culturales. Es imprescindible una nueva mentalidad o metodología de trabajo en la gestión cultural para el abordaje de una mayor interdependencia entre contextos que nos obliga a la cooperación cultural internacional y a la concurrencia en los nuevos espacios geopolíticos.

Esperamos que las nuevas oportunidades del sector cultural nos estimulen a los formadores a un cambio de perspectiva evitando caer en situaciones inmovilistas tan corrientes en el ámbito académico. De esta manera vamos a intentar situar la formación de gestores culturales en una buena sintonía con la realidad profesional y social, condición indispensable para mantenerse activo en el campo de la cultura.

\section{Nota}

I. Se basan en el trabajo realizado en el Programa Format de la Inicitiva Adap por el Observatorio Interarts y la Diputación de Barcelona. http://www.interarts.net/format

\section{LA FORMACIÓN DE LOS GESTORES DE PATRIMONIO CULTURAL}

Francisco Zamora Baño

Presidente de la Asociación Española de Gestores de Patrimonio Cultural

La respuesta a la pregunta sobre qué formación es deseable para los gestores de patrimonio cultural requiere contestar previamente a otra pregunta, a saber, qué función se le exige a estos profesionales; es decir, pasa por definir el perfil tipo de un gestor profesional de patrimonio cultural.

Un gestor (en general y en el sentido que en nuestro sector ha venido a tener) no es sino un profesional que ha adquirido la metodología, las técnicas y los conocimientos adecuados para administrar eficientemente los recursos puestos a su disposición y ordenarlos a la consecución de los objetivos que se le hayan fijado previamente. Su currículo formativo corresponde al que se imparte en los estudios de dirección y administración de empresas, tanto de grado como de postgrado, y su actividad concreta se resume en el conocido pentálogo de la dirección de empresas: "prever, organizar, mandar, coordinar, controlar".

¿Qué es, entonces, lo específico del Gestor de Patrimonio Cultural? En el párrafo anterior nos hemos referido a los recursos puestos a disposición del gestor (en sentido general) para que, administrándolos adecuadamente, obtenga ciertos resultados: una plantilla de personal, un presupuesto económico, unos medios materiales, edificios, equipo informático, etc. Pues bien, lo específico del Gestor de Patrimonio Cultural es el hecho de que el principal recurso que se le encomienda es un bien de patrimonio cultural (un yacimiento arqueológico, un museo, un recinto histórico, un castillo, una ruta cultural, etc.), y que, entre los objetivos que se le fijan, el principal, explícita o implícitamente, es la salvaguardia de la integridad de dicho bien.

Por ello, en la Asociación Española de Gestores de Patrimonio Cultural definimos la Gestión de Patrimonio Cultural como "la eficiente administración de recursos (culturales, humanos, económicos y de todo tipo) ordenada a la consecución de objetivos sociales que afecten al patrimonio cultural". Igualmente, en nuestro Código Deontológico se destaca el principio ético de que "toda la actividad del Gestor de Patrimonio Cultural estará gobernada por un inmutable respeto hacia la significación histórica y estética y la integridad física de los elementos de Patrimonio Cultural cuya gestión se le confíe."

La sociedad actual pide al Gestor de Patrimonio Cultural que satisfaga una triple demanda. En primer lugar, que preserve el elemento de patrimonio cuya gestión se le haya confiado. La conservación del Patrimonio Cultural es una conditio sine qua non que debe primar ante todo, ya que una mala gestión puede poner 
en peligro elementos de patrimonio que son por su propio carácter irrepetibles. En segundo lugar, que optimice los recursos (humanos, económicos, materiales) que la sociedad ponga a su servicio. Es decir, que sea un eficiente administrador de esos recursos. $Y$, en tercer lugar, que obtenga rentabilidad social, es decir, cultural, y (si ello forma parte de sus objetivos) también económica del bien patrimonial que se le haya confiado, que maximice sus recursos hasta el punto que sea compatible con la conservación del bien, que, debemos repetir, debe primar ante todo.

O sea, que en conseguir al mismo tiempo asegurar la conservación del bien cultural, por una parte, con la administración eficiente de unos recursos, por otra, y con la obtención de una rentabilidad social, cultural y económica del bien, reside el "quid" del gestor de Patrimonio Cultural. No es una tarea fácil, y para ello el Gestor de Patrimonio Cultural necesita una amplísima formación que va desde un profundo conocimiento específico del bien cultural que ha de gestionar hasta múltiples y variados conocimientos técnicos procedentes, unos del campo de la Dirección de Empresas, otros de la Gestión Pública, además de mucha imaginación y no poca creatividad.

¿Cómo podemos formar gestores de patrimonio cultural que tengan esta enorme, pero necesaria, pluridisciplinariedad? No es fácil.

Supongamos un gestor al frente de un yacimiento arqueológico que se pretende poner en valor, con todos los recursos que ello conlleva. Es lógico que debe comprender a fondo la problemática cientíica del yacimiento, $y$, para ello, nada mejor que ser arqueólogo él mismo. Pero además, el resto de los recursos que debe administrar convierten al yacimiento en una verdadera empresa: presupuesto económico, recursos humanos, edificios, equipo, marketing, obtención de recursos, canalización de las visitas protegiendo el yacimiento, etc. Debe, además, ser capaz de diseñar y poner en práctica proyectos adecuados a la consecución de objetivos. Debe conocer la legislación estatal, autonómica e internacional que afecte a su trabajo ¿Un arqueólogo que sea también abogado y economista? Podría ser. ¿O un arqueólogo que haya hecho un Máster en Dirección de Empresas? También valdría. Luego, la experiencia, los años, y el reciclaje continuo pulirán este profesional hasta hacerlo perfectamente adecuado para su labor.

Es claro, sin embargo, que de esta manera hemos formado un profesional capaz, en principio, sólo para gestionar patrimonio arqueológico. Para otros tipos de patrimonio necesitaríamos, de arranque, otra formación básica. Historiadores del arte, arquitectos, restauradores y otra larga serie de profesionales darán la formación básica en Patrimonio Cultural según el bien de que se trate; todos ellos habrán de complementar su preparación como gestores con el otro aspecto de la formación que llamaremos, para entendernos, "empresarial."
¿Es posible, según lo que hemos dicho hasta ahora, formar un tipo de gestor de patrimonio cultural "generalista", es decir, uno que fuera adecuado para ponerlo al frente de cualquier empresa, institución u organismo relativo al patrimonio cultural? La respuesta ha de ser afirmativa si nos fijamos en los estudios de postgrado que, con parecidas denominaciones, son ofrecidos desde hace pocos años por varias universidades españolas. A estos cursos asisten licenciados de las más diversas carreras: historiadores del arte, licenciados en geografía e historia, en filosofía, en ciencias económicas, abogados, arqueólogos, arquitectos, licenciados en bellas artes, y muchas más. El currículo de estos estudios, que incluye tanto materias formativas en patrimonio (llamémosle la "parte artística") como materias y técnicas de "gestión empresarial", trata de rellenar las carencias de la doble formación a que nos referíamos antes.

El campo de la gestión de patrimonio cultural es, como se sabe, enormemente variado. Gestionar un museo no es lo mismo que diseñar una ruta histórica o dirigir una empresa de restauración artística o crear un centro de interpretación. Cada uno de las decenas de tipos diferentes de trabajos que caben en la gestión de patrimonio cultural requeriría una formación específica. Pero ello pasa en todas las profesiones: en el derecho, en la medicina o en el arte. Hay que admitir que una formación generalista en gestión de patrimonio cultural es simplemente un primer paso que faculta a estos titulados a iniciar su carrera en el sector. Cada uno de los diferentes puestos que desempeñen les demandarán aprender algo nuevo, demanda que llenarán con el estudio, la formación continua y la experiencia. Al final, será inevitable para muchos un grado mayor o menor de especialización, aunque también hay y habrá empresas que tengan los medios y la capacitación para acometer un amplio abanico de temas diferentes.

Lo que creo que está claro, para terminar, es que, con todas las dificultades que el tema formativo conlleva, el patrimonio cultural, y más en un país como el nuestro, es algo lo suficientemente importante como para que su gestión deba confiarse a profesionales preparados específicamente para ello, al margen de improvisaciones y voluntarismos. Esta es una idea que ya ha penetrado en la sociedad y la universidad ha recogido el guante. Esperamos que penetre igualmente en las diversas administraciones públicas a las que las leyes han confiado esta valiosa herencia.

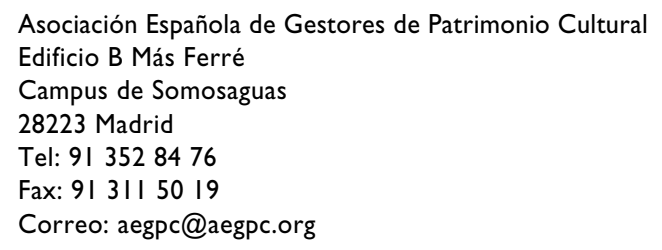

Asociación Española de Gestores de Patrimonio Cultural 


\section{LA ASOCIACIÓN DE GESTORES CULTURALES DE ANDALUCÍA (GECA) Y LA FORMACIÓN}

Francisco Javier Rodríguez Fito Presidente de la GECA

La sociedad andaluza ha sufrido en los últimos tiempos una transformación considerable, en parte por impulso de las instituciones democráticas, en parte por el esfuerzo de ella misma. Dentro de los cambios que pueden apreciarse a simple vista es destacable el papel que el hecho cultural desempeña en nuestros días en la vida cotidiana, tanto en sus aspectos sociales como en los económicos. Cuando las administraciones públicas se plantean la intervención en lo cultural promueven, a veces casi sin quererlo, la aparición de un grupo de nuevos profesionales cuya labor principal es el trabajo en el ámbito de la cultura. Estos trabajadores reciben diversas denominaciones: animadores socioculturales, gestores, técnicos de cultura, etc. Poco a poco su número aumenta, pero la definición de sus funciones, del papel que les corresponde en el entramado administrativo, la estructura profesional básica que les defina, sigue sin estar clara. En el día de hoy para muchos de estos profesionales, para las instituciones, para los sectores creativos y para la sociedad en su conjunto no está claro qué elementos, qué características diferencian a un Gestor Cultural de un mero organizador de actividades culturales o de ocio. Si unimos a esto la diversidad de modelos de contratación, de categorías profesionales, de organigramas de funcionamiento y de formas de relación con el poder político, entre otros asuntos, nos encontramos con una realidad compleja y frustrante que puede conducir a un bloqueo o parálisis de la intervención pública en el ámbito de la Cultura.

Para responder a esta compleja situación nace la GECA. La Asociación trata de agrupar a todos los profesionales de nuestra comunidad autónoma para la definición de su papel en el conjunto de la Andalucía actual. Porque entendemos que la cultura en una sociedad como la nuestra no puede ser algo gratuito y decorativo, sino que ha de transformarse en un elemento de apoyo a los procesos de modernización y desarrollo. Ordenar nuestra profesión, situarla en el entramado social, reflexionar sobre el hecho cultural, dialogar con los sectores creativos y con la iniciativa social, éstos son los objetivos primordiales de la Asociación GECA, que nace en estos tiempos de crisis y de necesario esfuerzo para todos.
Los fines de la Asociación son:

- La definición del ámbito profesional de los gestores de cultura.

- La investigación y análisis de todo lo referente al acceso y ejercicio de dicha profesión.

- El estudio, búsqueda y ejecución de cuantas propuestas vayan encaminadas a mejorar tanto la formación como la cualificación de sus miembros y su consideración profesional.

- Elaborar propuestas para mejorar la articulación entre los sectores institucionales, profesionales, creativos y sociales que intervienen en el hecho cultural.

- Todos aquellos fines que ética y profesionalmente redunden en el beneficio de sus asociados.

- El fomento de la cultura como parte esencial del desarrollo local, como fuente de empleo y garantía de bienestar social.

- La atención de manera especial a los conceptos de identidad cultural y territorio.

La GECA es consciente de que una de las herramientas fundamentales para conseguir los fines propuesto es la formación de los profesionales de la gestión cultural. Para ello pretende organizar directamente, o en colaboración con otras instituciones, cursos de especialización en las distintas temáticas de interés para los profesionales de la gestión cultural en Andalucía.

En esta línea está previsto ofertar en diciembre del 2000 un primer curso sobre Marketing de servicios culturales a impartir en Sevilla, en colaboración con el Instituto Andaluz del Patrimonio Histórico.

\section{Información:}

Asociación de Gestores Culturales de Andalucía GECA c/ Cervantes, 4

Bellavista. 21 122 - Huelva.

TIf.: 677433066

Dossier elaborado por Pilar Tassara Andrade Dpto. de Formación, IAPH 